\title{
Source Inversion of the W-Phase: Real- time Implementation and Extension to Low Magnitudes
}

\section{Gavin P. Hayes, ${ }^{1}$ Luis Rivera, ${ }^{2}$ and Hiroo Kanamori ${ }^{3}$}

Online material: Results for $W$-phase inversions for 498 events with $M \geq 5.8$ in 2007-2008, compared to results from the global Centroid Moment Tensor catalog.

\section{INTRODUCTION}

We assess the use and reliability of a source inversion of the $W$-phase in real-time operations at the U.S. Geological Survey National Earthquake Information Center. The three-stage inversion algorithm produces rapid and reliable estimates of moment magnitude and source mechanism for events larger than $M_{w} 7.0$ within 25 minutes of the earthquake origin time, often less, and holds great promise for vastly improving our response times to such earthquakes worldwide. The method also produces stable results (within \pm 0.2 units of Global Centroid Moment Tensor project estimates) for earthquakes as small as $M_{w} 5.8$ when using stations out to distances of $90^{\circ}$. These applications extend the use of $W$-phase far beyond the higher magnitude events for which the inversion was originally intended, facilitating its use as a complementary approach to traditional body- and surface-wave methods for assessing the source properties of an earthquake.

Kanamori and Rivera (2008) introduced the use of $W$-phase as a reliable method to rapidly assess the source properties $\left(M_{w}\right.$ and mechanism) of earthquakes greater than $\sim M_{w}$ 7.5. They showed that the $W$-phase inversion method has important real-time applications for tsunami warning purposes (and indeed for the calculation of earthquake moment), particularly for very large earthquakes such as the 2004 SumatraAndaman Islands event for which traditional methods may suffer due to the clipping of seismograms and/or because they do not deliver accurate results quickly enough to be useful for tsunami warning.

1. U.S. Geological Survey, National Earthquake Information Center (contracted by Synergetics, Inc.), Golden, Colorado

2. Institut de Physique du Globe de Strasbourg, France

3. Seismological Laboratory, California Institute of Technology, Pasadena, California, U.S.A.
The U.S. Geological Survey (USGS) National Earthquake Information Center (NEIC) began implementation of $W$-phase inversion in real-time operations for testing purposes in July 2008. Here we assess the performance of this method in real time and show that $W$-phase not only provides rapid and accurate results for large earthquakes but also that this inversion can be applied successfully to earthquakes as small as $M_{w} \sim 5.8$. Such applications extend the magnitude range of the inversion far beyond that from which accurate results were previously expected $\left(M_{w}>\sim 7.0\right)$ and facilitate the use of this method as a new moment tensor inversion algorithm for most damaging earthquakes worldwide.

\section{W-PHASE}

For information on the details of the wave-theory and modeling of $W$-phase, we refer readers to Kanamori and Rivera (2008); here we give just a brief overview. The $W$-phase is a long-period (approximately 100-1,000 s) phase arriving between the $P$-and $S$-wave phases of a seismic source, theoretically representing the total near- and far-field long-period wave-field. For modeling purposes, $W$-phase can be synthesized by a summation of normal modes (fundamental, 1st, 2nd, and 3rd overtones). We follow the inversion procedure described by Kanamori and Rivera (2008), altering the frequency band depending on the initial estimate of earthquake magnitude (Table 1). Over the short-period bands used for inversion of the smaller earthquakes $\left(\sim M_{w}<7\right), W$-phase includes the energy carried mainly by body-wave phases such as $P, P P, P P P, P S, S P, S$, and $S S$ arriving within the time window used (from $P$-wave arrival time to $15 \Delta$ s, where delta is distance in degrees) and is thus most conveniently interpreted as a superposition of these body waves. Over the longer-period bands used for large earthquakes modeled here and in previous applications of $W$-phase inversions, $W$-phase can be viewed as a normal mode superposition.

Currently, the initial magnitude estimate used by the inversion scheme comes from preliminary evaluations performed by the NEIC or one of the two National Oceanic and Atmospheric Administration (NOAA) tsunami warning cen- 


\begin{tabular}{|ccc|}
\hline \multicolumn{3}{|c|}{ TABLE 1 } \\
$\begin{array}{c}\text { Corner Frequencies Used for Butterworth Bandpass } \\
\text { Filtering (1 pass, 4th order) in } \boldsymbol{W} \text {-phase Inversion, Based } \\
\text { on Initial Magnitude Estimates }\end{array}$ \\
\hline $\begin{array}{c}\text { Magnitude Range } \\
\left(\boldsymbol{M}_{w}\right)\end{array}$ & $\begin{array}{c}\text { Low Corner } \\
\text { (Hz) (s) }\end{array}$ & $\begin{array}{c}\text { High Corner } \\
\text { (Hz) (s) }\end{array}$ \\
\hline$M_{w}>8.0$ & $0.001(1000 \mathrm{~s})$ & $0.005(200 \mathrm{~s})$ \\
$8.0>M_{w} \geq 7.5$ & $0.00167(600 \mathrm{~s})$ & $0.005(200 \mathrm{~s})$ \\
$7.5>M_{w} \geq 7.0$ & $0.00167(600 \mathrm{~s})$ & $0.01(100 \mathrm{~s})$ \\
$7.0>M_{w}>6.5$ & $0.002(500 \mathrm{~s})$ & $0.01(100 \mathrm{~s})$ \\
$6.5 \geq M_{w}$ & $0.0067(150 \mathrm{~s})$ & $0.02(50 \mathrm{~s})$ \\
\hline
\end{tabular}

ters and usually corresponds to an $M_{w} \mathrm{p}$ magnitude (Tsuboi $e t$ al. 1995). Green's functions (computed via normal-mode summation) are stored in a separate database, precomputed every $0.2^{\circ}$ between $0^{\circ} \leq \Delta \leq 90^{\circ}$ over a depth range of $0-760 \mathrm{~km}$. Though in practice $W$-phase inversion is most appropriately performed with LH channel data (1 sample-per-second, sps), we use broadband data resampled to 1 sps to avoid time delays in gathering real-time data arising from data latency.

The real-time application of the $W$-phase inversion at the NEIC involves three main stages. Initially, the inversion uses the NEIC Preliminary Determinations of Epicenters (PDE) hypocenter and estimates of centroid time shift $\left(t_{d}\right)$ and half duration $\left(t_{h}\right)$ based on the initial NEIC/NOAA magnitude (Kanamori and Rivera 2008). In the second stage, we apply a 1-D grid search for $t_{d}$ by minimizing the root mean square (RMS) of the waveform misfit. With this optimized time-shift, a second inversion updates the initial solution. In the third and final stage, we perform another grid search for the optimal centroid location of the event over a $\pm 2^{\circ}$ area centered on the initial hypocenter, sampled every $0.5^{\circ}$. This initial grid size is increased (in both grid searches) if the solution is within one cell of the grid edge. A third inversion with these optimized parameters yields our final solution. An example of this threestage approach and the results at each stage are shown in Figure 1 for the 2009/01/03 $M_{w} 7.6$ Papua earthquake, computed with stations to a distance of $\Delta=90^{\circ}$. Waveform fits for the last stage at a selection of stations are shown in Figure 2.

\section{EXAMPLES—LARGE EARTHOUAKES}

Since the real-time implementation of the $W$-phase inversion at the NEIC in mid-July 2008, there have been five earthquakes of $M_{w}>7.0$. In Figure 3 we show results for these five events computed with 1) stations from $10^{\circ} \leq \Delta \leq 50^{\circ}$ and 2) stations from $10^{\circ} \leq \Delta \leq 90^{\circ}$. The solutions shown here were produced at the third stage of the inversion process, after the optimized $W$-phase centroid grid search. These results are compared to solutions for the same events from the Global Centroid Moment Tensor project (GCMT, http://www.globalcmt.org; Ekström et al. 2005).

All inversions yield very good results. Magnitude estimates are within \pm 0.1 units when compared to GCMT results, and mechanisms are very similar, though slightly rotated for two

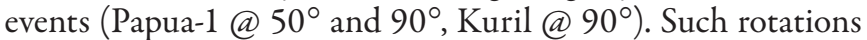
are likely caused by differences in centroid locations between the two inversions. In real-time applications, our $W$-phase centroid location grid search is performed on a grid with spacing of $0.5^{\circ}$ (Figure 1). We note that for the first Papua and Kuril events, if we decrease this spacing to $0.1^{\circ}$, results improve with respect to the GCMT solution (Figure 3). In real-time operations, solutions with stations to $\Delta=50^{\circ}$ are available on average approximately 24 minutes after the event origin time $(\mathrm{OT})$; 48 minutes after OT with stations to $\Delta=90^{\circ}$.

For the second large event north of Papua, Indonesia, on 2009/01/03, data were contaminated with noise from the earlier, larger event. In such cases, the true $W$-phase inversion does not produce reliable results in real time. However, we can simulate a CMT-style inversion by shifting the time-window of the inversion to encompass the surface waves and by adjusting the bandpass filter accordingly. Results in Figure 3 show such an approach is successful-the inversion yields good results, within 0.1 magnitude units of GCMT, and with a similar mechanism and centroid location. Though these adjustments are not currently part of the real-time operations at the NEIC, implementation would require only a straightforward initial assessment of recent inversion solutions and pre-event noise.

\section{Examples-Small Earthquakes}

Of 592 events with $\mathbf{M} \geq 5.8$ in the PDE catalog in 2007 and 2008, 550 also had solutions in the GCMT catalog, making them suitable for $W$-phase inversion comparisons and reliability assessments. Of these 550 events, inversions for 493 events produced $W$-phase magnitudes within \pm 0.2 units of the GCMT moment magnitude. Twenty-six inversions were aborted because no stations passed the inversion signal-tonoise screening filters, and 26 other solutions were considered of low quality as a result of the number of stations used in the inversion and the final condition number of the solution. This leaves just five events for which the difference between $W$-phase magnitude and GCMT magnitude is greater than 0.2 units. Each of these had $W$-phase magnitudes within \pm 0.3 units of the GCMT magnitude.

For one of these five events (the 2007/09/12 23:49 $M_{w} 7.87$ southern Sumatra earthquake; $W$-phase $\left.M_{w}\left(M_{w} w\right)=8.12\right)$ data were contaminated with noise from an $M_{w} 8.4$ earthquake 12 hours earlier. For three others $\left(2007 / 08 / 0300: 41 M_{w} 5.89\right.$, $M_{w} w 6.17 ; 2007 / 12 / 1608: 09 M_{w} 6.71, M_{w} w 6.46 ; 2008 / 07 / 19$ 09:27 $M_{w} 6.57, M_{w} w 6.85$ ), magnitude differences may have been caused by large shifts in $W$-phase centroid locations relative to the PDE hypocenter and/or GCMT centroid- $W$ phase magnitudes after the $t_{d}$ grid search were within \pm 0.2 units of the GCMT magnitude, and the centroid search shifted the events a distance greater than their probable source dimensions. The one remaining event $\left(2007 / 09 / 01,19: 14 M_{w} 6.12\right.$ Gulf of California) produced a $W$-phase magnitude of $M_{w} w$ 5.85 , a difference that may have been caused by a concentration of station coverage near the nodal planes of this strike-slip 


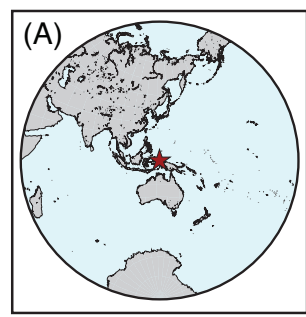

(B)

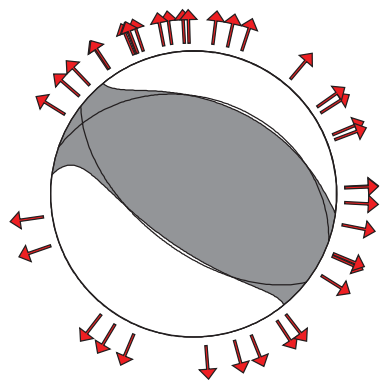

38 Stations

$t_{h}=18.9 \mathrm{~s}, t_{d}=16.4 \mathrm{~s}$

$M_{w}=7.57$

(C)

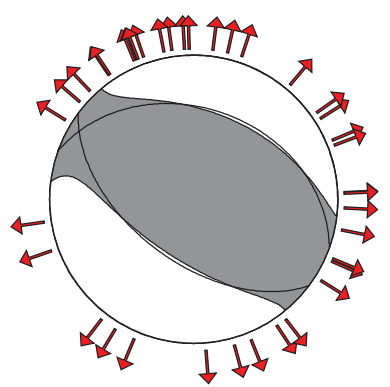

38 Stations

$\mathrm{t}_{\mathrm{h}}=\mathrm{t}_{\mathrm{d}}=12.0 \mathrm{~s}$

$M_{w}=7.55$

(D)

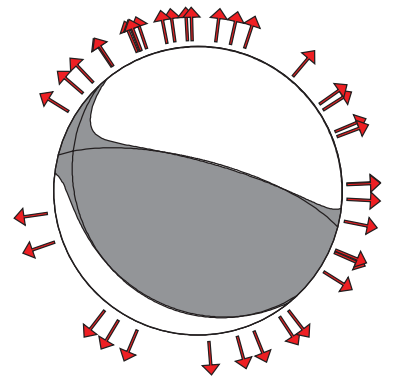

38 Stations

$\mathrm{t}_{\mathrm{h}}=\mathrm{t}_{\mathrm{d}}=12.0 \mathrm{~s}$

$\mathrm{M}_{\mathrm{w}}=7.56$

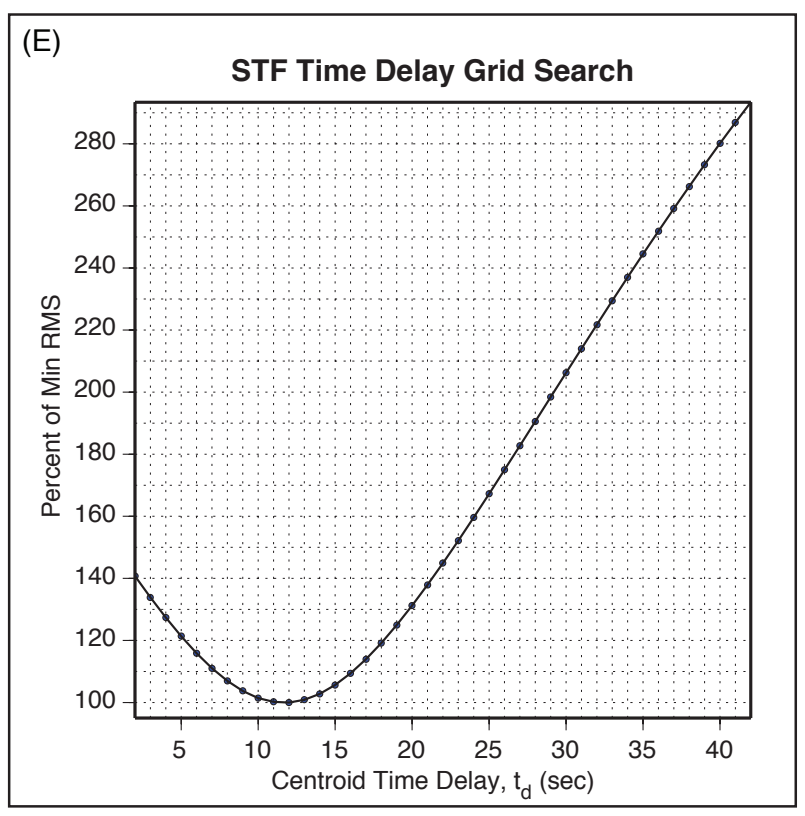

(F) Centroid Grid Search

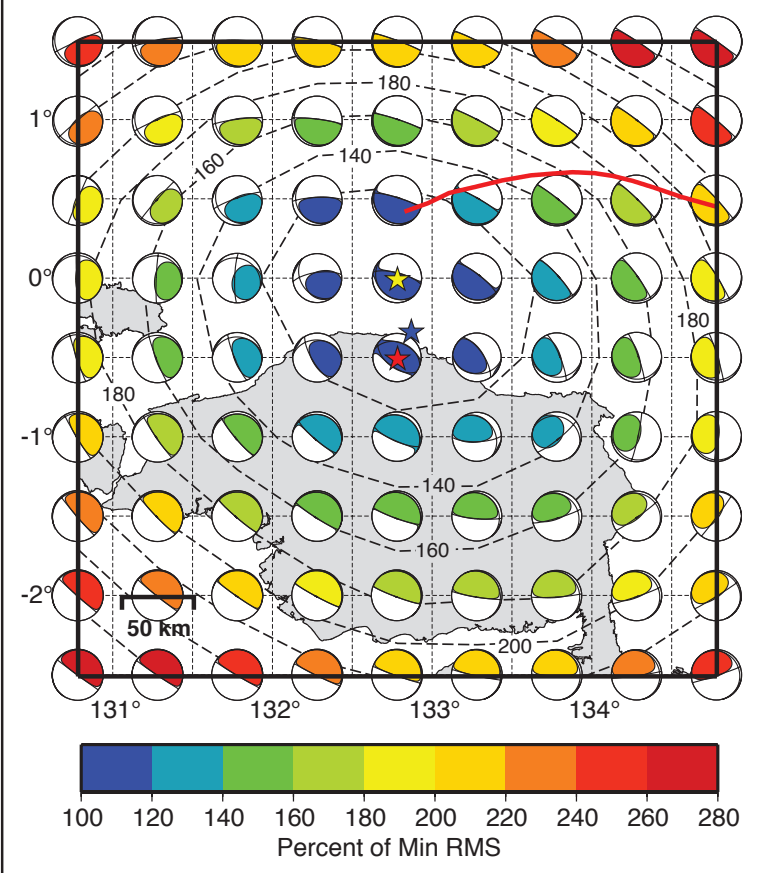

A Figure 1. Typical stages in a real-time $W$-phase inversion at the NEIC. (A) Results are shown for the 2009/01/03 $M_{w} 7.6$ Papua earthquake, indicated with a red star. (B) Inversion results using the initial PDE hypocenter and estimates of centroid time-shift and half-duration based on the initial NEIC magnitude. Red arrows around the circumference of the CMT indicate back-azimuths to stations used in the inversion. (C) Inversion results after a 1-D grid search for the centroid time-shift. (D) Inversion results after a grid-search for the optimized centroid location. (E) Results of the centroid time-shift grid search, where misfit is shown relative to the minimum RMS value. (F) Results of the centroid location grid search. Inversions are computed every $0.5^{\circ}$. Inverted CMTs are colored by their misfit percentage relative to the minimum RMS value. The best-fitting solution is shown with a yellow star. The red star indicates the initial PDE epicentral location and the blue star the GCMT centroid location. Dashed contours indicate the spatial misfit variability. Major plate boundaries are shown with thick red lines. 

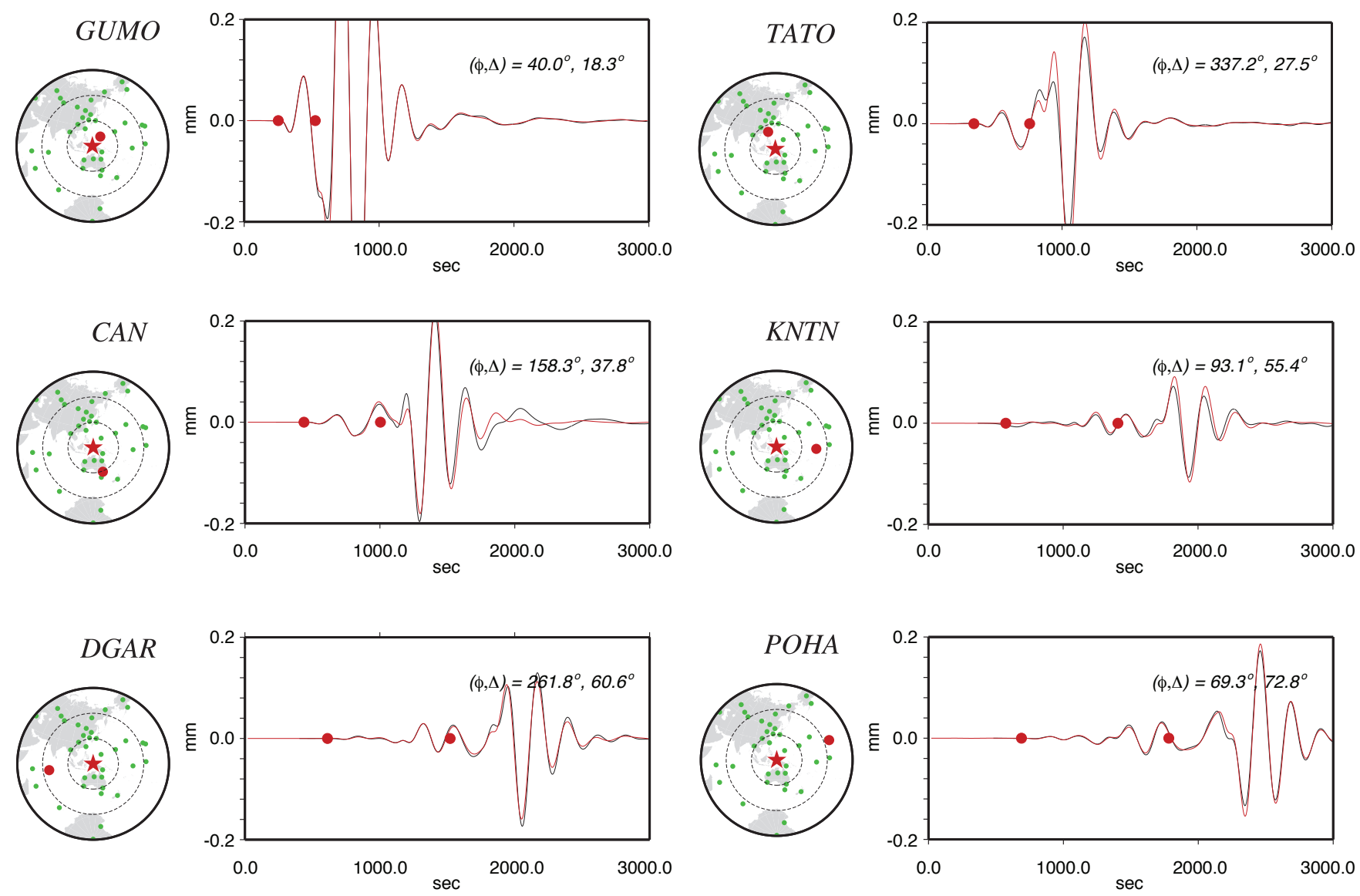

A Figure 2. Comparison of observed (black) and synthetic (red) waveforms at a selection of stations for the 2009/01/03 $M_{w} 7.6$ Papua earthquake summarized in Figure 1. The two red dots on each trace indicate the time window over which the $W$-phase is inverted.

solution. With the exception of the 2007/09/12 event, magnitude differences for all five of these events are reduced to within \pm 0.2 units of the GCMT magnitude when the grid spacing of the centroid search is reduced to $0.1^{\circ}$, on the order of the source dimensions for an $\mathbf{M} 6$ earthquake.

The results of these inversions are summarized in Figure 4 and in the online supplementary material and show that the $W$-phase inversion can be successfully implemented down to at least a magnitude of $M_{w} 5.8$.

\section{CONCLUSIONS}

The $W$-phase inversion method proposed by Kanamori and Rivera (2008) has been successfully implemented at the NEIC. Over the past seven months for which the inversion has been in operation, it has produced rapid and reliable magnitudes and source mechanisms for all earthquakes of $M_{w}>7.0$. Because of the fast group velocities of the $W$-phase and its insensitivities to lateral heterogeneities in the Earth, stable results for such large events can be obtained within $\sim 20$ minutes of the earthquakes' occurrence, making the inversion method highly valuable for rapid earthquake response.

Currently, the NEIC runs two moment-tensor inversions as part of its real-time operations: a body-wave moment tensor algorithm based on Sipkin (1982), which uses long-period (<
80 s) teleseismic data ( $P, S H$, and $S V$ phases) and is accurate over the approximate magnitude range $5.5 \leq M_{w} \leq 7.0$ (depending on the complexity of the source); and a centroid moment tensor algorithm based on Dziewoński and Woodhouse (1983), which uses 100-400 s data (dominated by Love and Rayleigh waves) and is accurate over the approximate magnitude range $6.0 \leq M_{w} \leq 8.5$. For near-source stations (within $10^{\circ}$ ), and for very large earthquakes $(\mathbf{M}>9)$, most moment tensor inversion methods suffer from clipped records; the $W$-phase inversion may be the only approach that can satisfactorily deal with such situations. As with the $W$-phase algorithm, speed of the NEIC inversions is predominantly controlled by the travel-times of the associated phases and by the station distribution surrounding the event; in general, accurate results can be obtained within 20-30 minutes for the body-wave inversion and 50-60 minutes for the surface-wave algorithm, given an earthquake of sufficient size. These times can often be prolonged if significant user input (data selection and filtering) is required.

We have shown that the $W$-phase inversion method can be successfully applied to earthquakes as small as $M_{w} 5.8$ when using stations within $\Delta=90^{\circ}$. Such applications extend the usefulness of $W$-phase beyond the realm of very large $\left(\sim M_{w} \geq 7.5\right)$ earthquakes and provide a complementary approach for earthquake source inversion alongside other more established bodyand surface-wave methods. $\mathbf{z}$ 


\begin{tabular}{|c|c|c|c|c|}
\hline & $\begin{array}{c}\text { W-Phase, } 50^{\circ} \\
\text { Optimized Centroid }\end{array}$ & $\begin{array}{c}\text { W-Phase, } 90^{\circ} \\
\text { Optimized Centroid }\end{array}$ & gCMT Solution & $\begin{array}{c}\text { W-Phase, } 90^{\circ} \\
\text { Optimized Centroid } \\
\text { Denser Grid Search }\end{array}$ \\
\hline $\begin{array}{c}\text { 2008/11/16 } \\
\text { Minahasa, Indonesia }\end{array}$ & & & & \\
\hline $\begin{array}{l}\text { Dist., (3)-(1) } 28 \mathrm{~km} \\
\text { Dist., (3)-(2) 28 km }\end{array}$ & $\begin{array}{c}11 \text { Stations } \\
t_{h}=t_{d}=14.2 s \\
M_{w}=7.31\end{array}$ & $\begin{array}{c}37 \text { Stations } \\
\mathrm{t}_{\mathrm{h}}=\mathrm{t}_{\mathrm{d}}=11.4 \mathrm{~s} \\
\mathrm{M}_{\mathrm{w}}=7.38\end{array}$ & $\begin{array}{c}\mathrm{t}_{\mathrm{d}}=12.3 \mathrm{~s}, \mathrm{t}_{\mathrm{h}}=11.0 \mathrm{~s} \\
\mathrm{M}_{\mathrm{w}}=7.3\end{array}$ & \\
\hline $\begin{array}{c}2008 / 11 / 24 \\
\text { Sea of Okhotsk }\end{array}$ & & & & \\
\hline $\begin{array}{l}\text { Dist., (3)-(1) } \sim 14 \mathrm{~km} \\
\text { Dist., (3)-(2) } \sim 14 \mathrm{~km}\end{array}$ & & $\begin{array}{l}\text { ations } \\
=5.2 \mathrm{~s} \\
7.28\end{array}$ & $\begin{array}{c}\mathrm{t}_{\mathrm{d}}=11.9 \mathrm{~s}, \mathrm{t}_{\mathrm{h}}=10.8 \mathrm{~s} \\
\mathrm{M}_{\mathrm{w}}=7.3\end{array}$ & \\
\hline $\begin{array}{c}\text { 2009/01/03, 19:43 PM } \\
\text { Papua, Indonesia }\end{array}$ & & & & \\
\hline $\begin{array}{l}\text { Dist., (3)-(1) } \sim 59 \mathrm{~km} \\
\text { Dist., (3)-(2) } \sim 50 \mathrm{~km} \\
\text { Dist., (3)-(4) } \sim 11 \mathrm{~km}\end{array}$ & $\begin{array}{c}19 \text { Stations } \\
t_{h}=t_{d}=10.1 \mathrm{~s} \\
M_{w}=7.52\end{array}$ & $\begin{array}{c}38 \text { Stations } \\
t_{h}=t_{d}=12.0 \mathrm{~s} \\
M_{w}=7.56\end{array}$ & $\begin{array}{c}\mathrm{t}_{\mathrm{d}}=15.4 \mathrm{~s}, \mathrm{t}_{\mathrm{h}}=15.9 \mathrm{~s} \\
\mathrm{M}_{\mathrm{w}}=7.6\end{array}$ & $\begin{array}{c}38 \text { Stations } \\
t_{h}=t_{d}=20.0 \mathrm{~s} \\
M_{w}=7.59\end{array}$ \\
\hline $\begin{array}{l}\text { 2009/01/03, 22:33 PM } \\
\text { Papua, Indonesia }\end{array}$ & & & & \\
\hline $\begin{array}{l}\text { Dist., (3)-(1) } \sim 27 \mathrm{~km} \\
\text { Dist., (3)-(2) } \sim 27 \mathrm{~km}\end{array}$ & $\begin{array}{c}19 \text { Stations } \\
t_{d}=4.4 \mathrm{~s} \\
M_{w}=7.39\end{array}$ & $\begin{array}{c}30 \text { Stations } \\
t_{h}=t_{d}=6.0 \mathrm{~s} \\
M_{w}=7.36\end{array}$ & $\begin{array}{r}t_{d}=3.5 \mathrm{~s}, t \\
\mathrm{M}_{\mathrm{w}}=\end{array}$ & \\
\hline $\begin{array}{l}\text { 2009/01/15 } \\
\text { Kuril Islands }\end{array}$ & & & & \\
\hline $\begin{array}{l}\text { Dist., (3)-(1) } \sim 23 \mathrm{~km} \\
\text { Dist., (3)-(2) } \sim 45 \mathrm{~km} \\
\text { Dist., (3)-(4) } \sim 26 \mathrm{~km}\end{array}$ & $\begin{array}{c}11 \text { Stations } \\
t_{h}=t_{d}=10.1 \mathrm{~s} \\
M_{w}=7.41\end{array}$ & $\begin{array}{c}43 \text { Stations } \\
t_{h}=t_{d}=8.3 \mathrm{~s} \\
M_{w}=7.40\end{array}$ & $\begin{array}{c}t_{d}=9.8 s, t_{h}=12.0 \mathrm{~s} \\
M_{w}=7.4\end{array}$ & $\begin{array}{c}43 \text { Stations } \\
t_{h}=t_{d}=8.3 \mathrm{~s} \\
M_{w}=7.39\end{array}$ \\
\hline
\end{tabular}

A Figure 3. $W$-phase inversion results for all $M_{w}>7.0$ events since real-time implementation began at the NEIC. Column 2 shows inversion results using stations to $\Delta=50^{\circ}$, and Column 3 using stations to $\Delta=90^{\circ}$. Column 4 shows GCMT inversion results for comparison. Column 1 lists event date and location and the distances between respective centroid locations. With the exception of the second 2009/01/03 Papua event, all inversions were completely automated. Results with stations to $50^{\circ}$ were complete on average 24 minutes after origin time; results with stations to $90^{\circ}$ took 48 minutes. The inversion for the second Papua earthquake on 2009/01/03 used a manually adjusted time window $(15 \Delta s-30 \Delta s$, rather than $P$-time $-15 \Delta s)$ to circumvent problems arising due to noise from the larger event three hours earlier. Column 5, where present, repeats results for column 3 using a optimized centroid grid search spacing of $0.1^{\circ}$ rather than the standard $0.5^{\circ}$, showing that results can be further improved with a higher density grid search (at the cost of time to completion). 


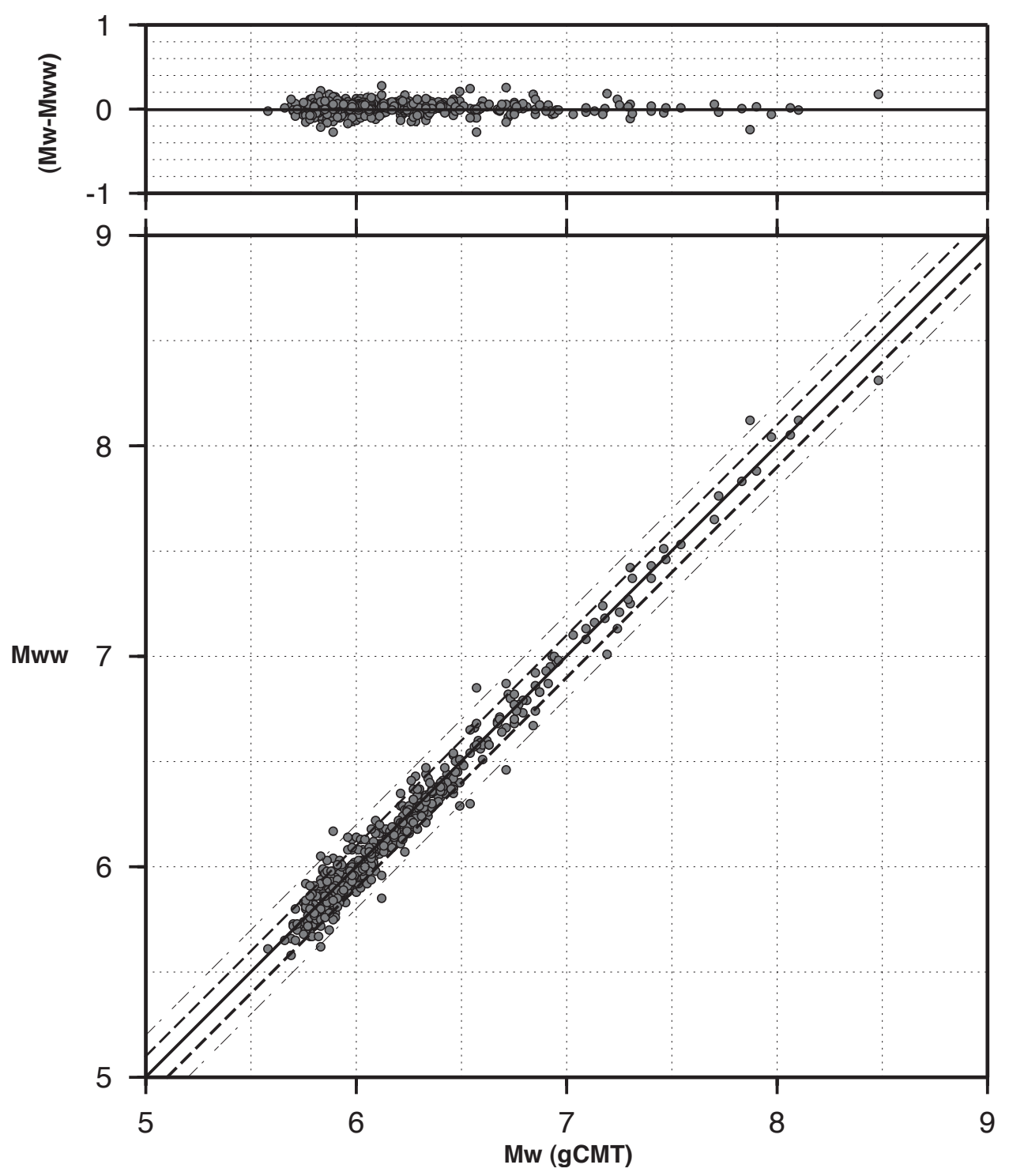

$\Delta$ Figure 4. Comparison of $M_{w}$ (GCMT) and $M_{w} w$ (W-phase) for 498 earthquakes of $M_{w} \geq 5.8$ in 2007 and 2008, computed with stations within $\Delta=90^{\circ}$. Ninety-nine percent of inversions with five stations above acceptable signal-to-noise ratios are within \pm 0.2 magnitude units. In both lower and upper panels, the solid black lines represents $M_{w}=M_{w} w$; in the lower panel, dashed lines indicate \pm 0.1 and dot-dash lines \pm 0.2 magnitude units.

\section{REFERENCES}

Dziewoński, A.M., and J. H. Woodhouse (1983). An experiment in systematic study of global seismicity: Centroid-moment-tensor solutions for 201 moderate and large earthquakes of 1981. Journal of Geophysical Research 88, 3,247-3,271.

Ekström, G., A. Dziewoński, N. Maternovskaya, and M. Nettles (2005). Global seismicity of 2003: Centroid-moment-tensor solutions for 1,087 earthquakes. Physics of the Earth and Planetary Interiors 148, $327-351$.

Kanamori, H., and L. Rivera (2008). Source inversion of $W$-phase: Speeding up seismic tsunami warning. Geophysical Journal International 175, 222-238.
Sipkin, S. (1982). Estimation of earthquake source parameters by the inversion of waveform data: Synthetic waveforms. Physics of the Earth and Planetary Interiors 30, 242-259.

Tsuboi, S., K. Abe, K. Takano, and Y. Yamanaka (1995). Rapid determination of $M w$ from broadband $P$ waveforms. Bulletin of the Seismological Society of America. 85, 606-613.

\section{U.S. Geological Survey National Earthquake Information Center P.O. Box 25046, MS 966 Denver, Colorado 80225 U.S.A. ghayes@usgs.gov} (G.P.H.) 